\title{
From Left Handed Materials to Invisible Cloak: Recent Advances
}

\author{
*Harihar Paudyala,d ${ }^{\mathrm{a},}$ Manoj Johrib,d and Akhilesh Tiwaric \\ aDepartment of Physics, Birendra Multiple Campus, Tribhuvan University, Bharatpur, Chitwan, Nepal, \\ ${ }^{b}$ Department of Physics and Electronics, DA-V College, CSJM University, Kanpur 208 001, India \\ 'Clermont Université, Université Blaise Pascal, Laboratoire de Génie Chimique et Biochimique (LGCB), BP 10488. \\ F-63000, France \\ ${ }^{\mathrm{d}}$ The Abdus Salam International Centre for Theoretical Physics, Strada Costeria 11, Trieste, Italy. \\ * hariharpaudyal@gmail.com
}

\begin{abstract}
Last decade has been increasing interest in metamaterial research, however metamaterials are sometimes regarded as left handed materials (LHM) or negative index materials but the recent progress in the field has shown that metamaterials are far beyond LHMs. In this paper we provide further advancement in the field of structured electromagnetic from LHM to invisible cloak.
\end{abstract}

Keywords: cloaking, negative index meta-material, photonic crystal.

\section{INTRODUCTION}

The first known [1-3] metamaterials (MMs) i.e. ruby glass with gold nano-particles embedded were used by artist and craftsmen for making art pieces centuries ago. The Lycurgus cup exhibited in the British museum and the Roman glass challis are famous examples (Fig. 1) exhibiting properties of these man-made materials with subwavelength inclusions. The scattering property of the material offered the cup a unique beauty, appearing green when viewed in reflected light such as daylight but reddish with light transmitted through the glass [4]. Scientists and engineers quest to build a microscope to glimpse a DNA with the naked eye and to create a cloak that makes an object invisible provoked them to fabricate structured and composite materials that either mimic known materials responses or qualitatively new physically realizable response functions that do not occur or may not be readily available in nature and in which controlled of light is not limited by the properties of optical materials that are existing, instead to create material that never were, by tailoring the elements of artificial structure down to the subwavelength scale. Effective medium theory, negative permittivity, negative permeability, Plasmon Polaritons, theory of periodic structures, resonant element small relative to the wavelength, frequency filters made of periodically arranged resonant elements, slow wave structure, superdirectivity super resolution, sub-wavelength focusing and imaging, inverse scattering, bianisotropy, photonic band gap material, waves on nanoparticals were alive at the time of the upsurge and could be regarded as various forerunners [5] of the subject of metamaterials. For MMs with negative permittivity and permeability several names and terminology have been suggested such as "Left Handed" media, "media with negative refractive index", "backward wave media", "double negative (DNG) metamaterials". LHM has unavoidable disadvantages of big loss and narrow band width and such disadvantages restricts the application of LHM while metamaterial has a much broader definition than LHM which does not require the negative permittivity or negative permeability and hence opens a completely new area. Metamaterials are typically constructed of "atoms" that have engineered electromagnetic response. The properties of the artificial atoms are often engineered to produce non-trivial values for the effective permittivity and effective permeability of lattice of identical atoms. Such value includes relative permittivities and permeabilities that are less than 1, close to zero, or negative. Traditional metamaterials utilizes wires to influence the dielectric properties by manipulating the effective plasma frequency of the medium [6]. The 
magnetic properties of split ring resonators (SRRs) are utilized to create a frequency band of subunit negative or near-zero magnetic permeability [7]. The sole of MMs is the ability to control electromagnetic waves. Further, one way to define MMs as artificially structured materials which attains its properties from the unit structure rather than the constituent materials [8]. MMs have an in-homogeneity scale that is much smaller than the wavelength of interest, and its electromagnetic response is expressed in terms of homogenized material parameters. Metamaterials are also considered as materials in either a thermodynamically meta-stable state (a shallow local minimum) or an unstable state (no minimum). The major challenge is to stabilize these metamaterials by kinetically slowing down their conversion to the stable thermodynamic phases. Most recently Johri and Paudyal [9] briefly discussed about technological impact of LHMs and their physical significance. (a)

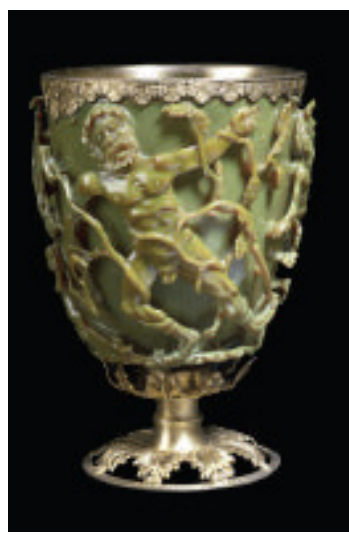

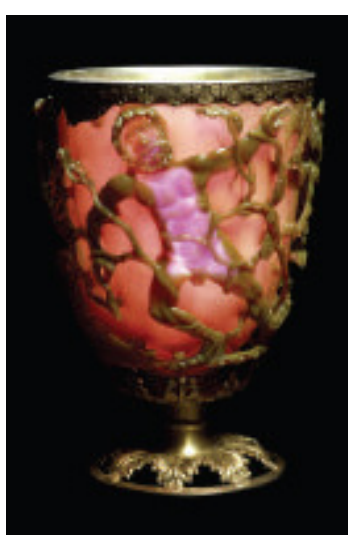

(b)
Figure 1 (a and b): The Lycurgus Cup 1958, 1202.1 in reflected (a) and transmitted (b) light. Scene showing Lycurgus being enmeshed by Ambrosia, now transformed into a vine-shoot. Department of Prehistory and Europe, The British Museum. (C) The Trustees of the British Museum [10]

The idea of a device which makes object invisible to the eye has a very long history, starting from folklore of many nationalities; we all have heard about various "invisibility hats" or "invisibility cloaks" such as the cloak of Harry Potter, main character in J K Rowling's novels, but can such a device be practically realized, at least in some limited frequency range? Can a finite size body be made invisible for electromagnetic radiation?
Scientists have been thinking for these questions for a long time. Cloaking and invisibility have, in recent years become facts instead of fiction, after the discovery and realization of various types of metamaterials and plasmonic materials. Many different techniques exist to obtain cloaking from electromagnetic or acoustic waves accordingly we define cloaking as a device which makes an object "invisible" for electromagnetic radiation in a certain frequency range [11]. In this paper we restrict ourselves only to recent advances and breakthroughs that this field experienced and unforeseen explosion at the beginning of the twenty-first century [12-14]. The article will be useful to the interested readers as stepping stone towards more advance research currently underway in the field.

\section{BRIEF HISTORY FROM LEFT HANDED MATERIAL TO INVISIBLE CLOAK}

The pursuit of artificial material for electromagnetic application is not new, this activity has a long history in modern science, first artificial electromagnetic metamaterial was engineered by J C Bose [15] when he worked and experimented on the constructed twisted elements (Fig. 2) that exhibit properties nowadays known as chiral characteristics. Horace Lamb [16] in 1904 and Henry Pocklington [17] in 1905 pointed out that mechanical system such as certain loaded chains can have the phase and group velocities in the opposite direction. Lindell [18] in 1914 studied artificially chiral media formed by an ensemble of small wire helices. Arthur Schuster [19] showed that this behavior could also be related in optical systems.

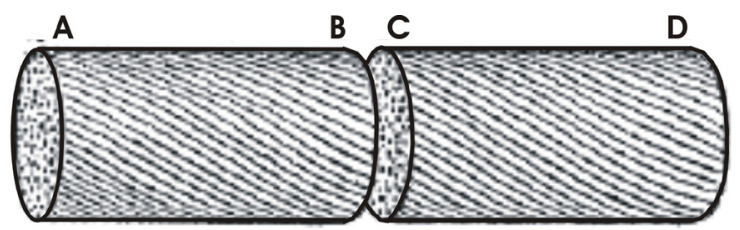

Figure 2: Twisted jute elements example of Chiral molecules [15]

Prof. Mandelstam from Moscow University in 1940 published a remarkable paper where he indicated the possibility of negative refraction caused by anti-parallel direction of group velocity and wave number of electromagnetic wave [20]. The negative 
refraction and backward wave media had been discovered theoretically much earlier [21-24]. In 1948 Kock [25] introduced the concept of the artificial dielectric in order to realize light weight lenses at microwave frequencies. Malyuzhinets in 1951 [23] studied Sommerfield radiation condition in backward wave media, he showed that phase velocity of waves pointed from infinity to source. In 1957 Sivukhin [24] published his work that showed the specific properties of a matter with a negative $\varepsilon$ and $\mu$. The inverted Doppler Effect, Cerenkov Effect in isotropic medium and negative refractive index were examined as early as in 1959 by Pafomov [26]. In 1960 backward wave structures had been developed for the design of microwave tubes [27-29]. Negative refraction was discovered even in periodical media [30]. Dollin (1961) described an inhomogeneous anisotropic magneto dielectric structure, such that a planer wave falling from infinity on this body "passes through it without distortion" [31]. These hypothetical media were systematically studied by Victor Veselago [32] in the 1968. As another example of early work on cloaking was of Kerker who published a paper entitle "invisible bodies" in 1975 [33]. Due to lack of experimental verification, research in LHMs was dormant for about three decades. In 1996, Pendry et al. [6] discovered the artificial electric plasma using the wire medium whose permittivity is negative. This work was followed by discovery of artificial magnetic plasma having negative permeability in 1999 [7]. In the mean time, Rodger M Walser at University of Texas at Austin synthesized the term of metamaterial and defined it as "microscopic composites having a synthetic three dimensional, periodic cellular architecture designed to produce an optimized combination, not available in nature of two or more responses to specific excitation [34]. In 2000 Smith, et al. [35] demonstrated that it is possible to fabricate an artificial material with electrodynamic characteristics that can be described by a negative index of refraction i.e. Negative Index Material or Left Handed Materials [8]. Shelby et al. [36] constructed a prism shaped section from such a metamaterial and their sample consists of square copper split ring resonators and copper wire strips on fiber glass circuit board material. The rings and wires are on opposite sides of the boards, and the boards have been cut and assembled into an interlocking lattice. They have been able to observe the negative refraction, as illustrated in Figures 2a and $2 b$.

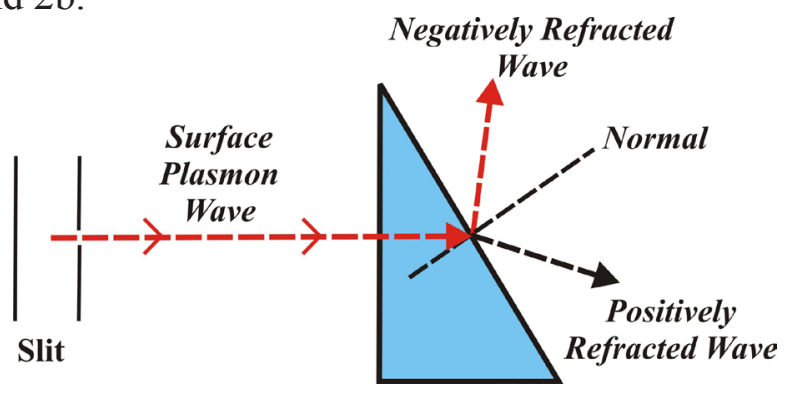

Figure 2a: Negative vs Positive refraction [37]

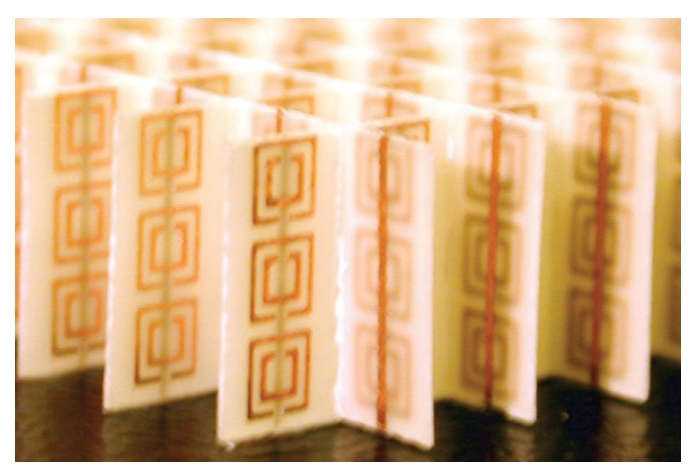

Figure 2b: Photograph of first experimentally realized the left handed metamaterial [36].

Negative refraction by a slab of materials bends a ray of light back toward the axis and thus has a focusing effect at the point where the refracted rays meet the axis (Fig. 3A)[38]. In 2000 Pendry [39] analyzed that a negative index lens exhibits an entirely new type of focusing phenomenon, bringing together not just the propagating rays but also the finer details of the electromagnetic near fields that are evanescent and do not propagate (Fig. 3B) [38].
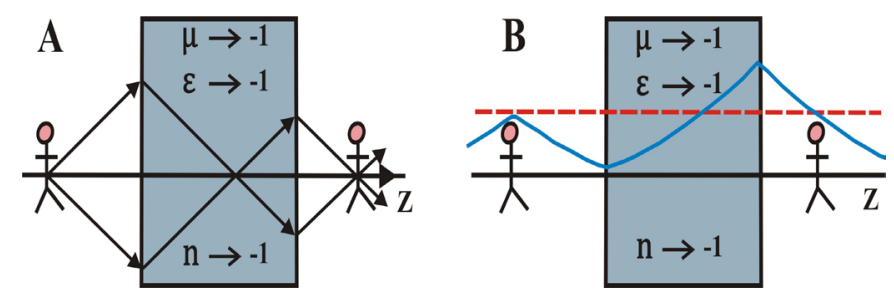

Figure 3: Perfect lensing in action: (A) the far field and (B) the near field using a slab of left handed material. 
For a planar slab of negative index material under idealized conditions an image plane exists that contains a perfect copy of an object placed on the opposite side of the slab. Although realizable materials will never meet the idealized conditions, nevertheless, these new negative index concepts show that wavelengths imaging is achievable. In 2004 Smith et al. [38] realized the gradient reflection index medium to bend electromagnetic waves. Pendry and his co-workers [40] proposed optical transformation to make invisible cloaks to control the propagation of electromagnetic waves using left handed materials in the microwave regime. Experimental realization of invisible cloak in the microwave regime $[41,42]$ and at optical frequencies [43-46] have been performed in two dimensional (2D) wave guide geometries. In other words these structures are immediately visible from the third dimension. Cloaking works only in the plane, the view angle is effectively zero in one direction nevertheless, these structures have supported the validity of the concepts both transformation optics and metamaterials. Recently Ergin et al. designed and realized a three dimensional invisibility cloaking structure operating at optical wavelength based on transformation optics [47] as visualized in Fig 4.

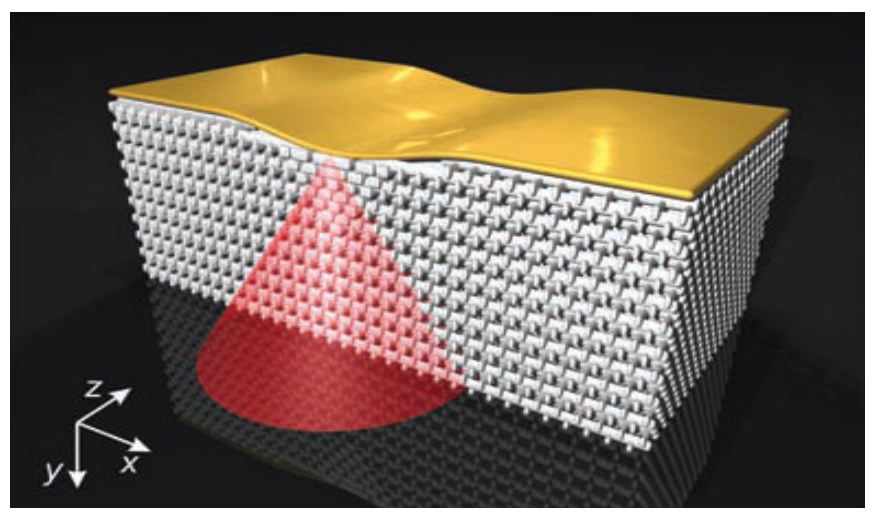

Figure 4: Blueprint of our 3D carpet-cloak structure [47].

Now the field has become a hot topic of scientific research and debate. A large number of publications are coming every year and it is not possible to cite all of them. We concentrate only on the pioneer developments which had a great impact on the physics, optics and engineering communities.

\section{OVERVIEW OF THE MATERIALS:}

Maxwell's equations resolve how electromagnetic waves propagate within a medium. The Maxwell's equation for a plane harmonic wave exp $[\mathrm{i}(k \cdot r-\omega t)]$ :

$$
k \times E=\omega \mu_{0} \mu H \text { and } k \times H=-\omega \varepsilon_{0} \varepsilon E,
$$

where $H$ and $E$ are the magnetic and electric fields respectively [48]. Taking a medium with negative real parts of $\varepsilon$ and $\mu$ with the imaginary parts being small at some frequency $(\omega)$, the vectors $H, E$ and $k$ will now form a left-handed harmony, which gives name for such materials as left-handed materials.

Metamaterials have much broader scope than LHM as shown in the Fig. 5 [49]. In the $\varepsilon-\mu$ region there are several different material properties such as the point $\mu=-\mu_{0}$ and $\varepsilon=-\varepsilon_{0}$ represent an anti-air in the LHM region, which will produce a perfect lens, the point $\mu=0$ and $\varepsilon=0$ represent a nihility, which can yield a perfect tunneling effect, the $\mu=\varepsilon$ in both RHM and LHM region represents impedance matching materials, which have perfect impedance matching with air, resulting no reflections. Also, the vicinity of $\mu=0$ is called $\mu-$ near zero material, and the vicinity of $\varepsilon=0$ is called $\varepsilon-$ near zero material which has special properties. The first quadrant $(\varepsilon>0, \mu>0)$ represents right handed electromagnetic propagating wave. Further Maxwell equation states that electric field $\mathrm{E}$, the magnetic field $\mathrm{H}$ and the wave vector $\mathrm{K}$ form a right handed system. While in the third quadrant $(\varepsilon<0, \mu<0)$ represents the backward propagating waves as proposed by Veselago [32] in the left handed material. At the same time in second $(\varepsilon<0$, $\mu>0)$ and in fourth $(\varepsilon>0, \mu<0)$ quadrant representing electric and magnetic plasma respectively which do not allow any propagating waves inside them i.e. supports propagation of evanescent waves. 


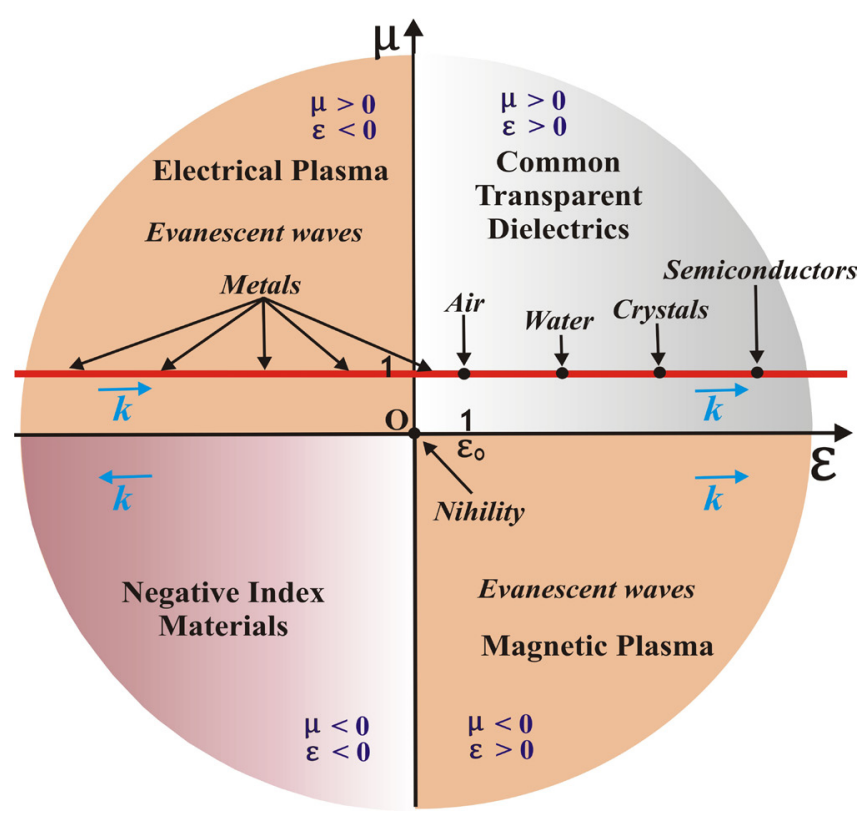

Figure 5: Overview of materials in the $\varepsilon-\mu$ domain. The two axes correspond to the real parts of permittivity and permeability respectively [49].

4. ELECTROMAGNETIC CLOAKING WITH METAMATERIALS:

The device that can hide an object from external electromagnetic radiation in a certain band of frequency is known as cloaking of course, the most exciting application is envisaged is working in the visible part of the spectrum. An object is invisible if it does not reflect waves back to the source and in addition, it does not scatter waves in other direction, and, furthermore, it does not create any shadow which means there is no scattering in the forward direction. From these conditions it follows that the object neither reflect nor absorb any power. In other words we can say the object should not disturb the fields existing outside the objects. More scientific way to explain cloaking [50] of electromagnetic wave is possible due to time invariant coordinate transformation of the governing Maxwell's equations. Such invariant transformation map a particular region in free space to spatial domain with position dependent and anisotropic material parameters (such as permeability and permittivity in electromagnetic), as in Figure 6.

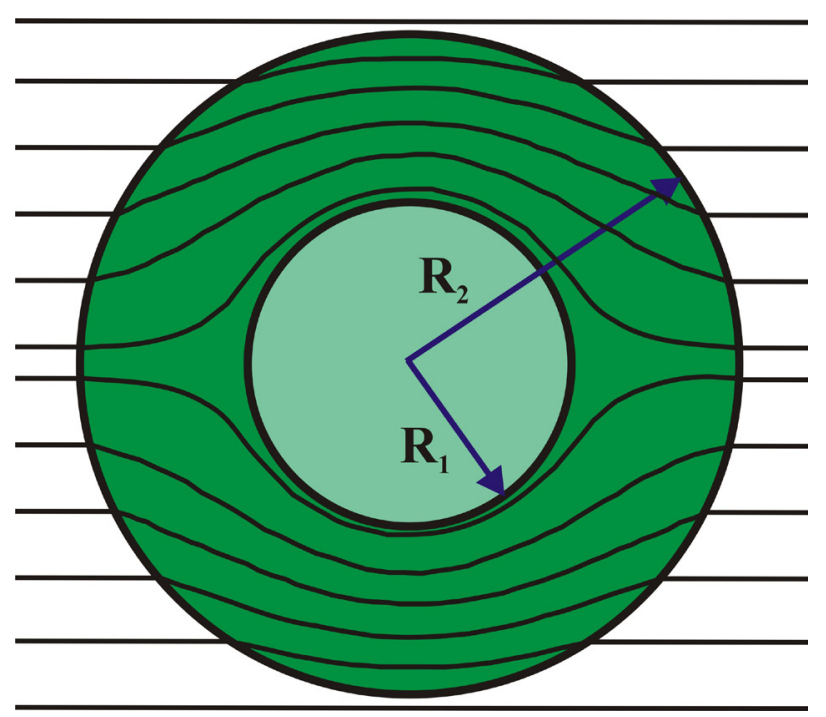

Figure 6: Optical cloaking with metamaterials: A two dimensional (2D) cross-section of rays striking the system, diverted within the annulus of cloaking material contained within $\mathrm{R}_{1}<\mathrm{r}<\mathrm{R}_{2}$ to emerge on the far side undeviated from their original course [40].

Cloaking should not be confused with the stealth technology. Stealth technologies minimize only the power reflected back to the probing radar (the back scattering cross section or "radar cross-section"). This can be done either by covering an object with an absorbing layer or by shaping the object so that the field scattered towards the illumination direction is minimized. Obviously, even an ideal stealth air craft invisible if observed from the side or from the back. It can be shown that absorbing coverings and object shaping cannot reduce the total scattering cross section by more than 50 percent. The concept of invisibility has been closely related to cloaking in recent literature [51]. One of the big challenges the realization of metamaterial cloak working for arbitrary polarization of the incident field is need to design the materials whose permittivity is equal to the permeability.

\section{APPLICATION:}

Recent advances made in the field of artificially structured materials from LHM to invisible cloaking can be expected to have numerous applications in near future but some of these are [52-59] in the near field technologies, photonic crystals [60-69] and plasmonics combined with innovation on high 
dielectric antennas, wireless systems and photonic circuits open the prospect of integrated circuits, sensors (chemical, biological and individual molecule), medical scanners, display and images which have the potential to outperform conventional technology and create many commercial opportunities. Some of the limitations that metallic lenses have such as imperfect matching with the free space can be overcome by these materials. The backward wave propagation in LHM with opposite direction of the group and phase velocities provides new applications including delay time filter and phase shifters from microwave to optical frequencies. Development and applications of metamaterials structures utilizing super conducting elements [70] due to their low loss, compact structure and nonlinear properties make them ideal candidates for realization of the landmark predictions of metamaterial theory including evanescent wave amplification, transformation optics, illusion optics, energy storage devices, parametric amplification of negative index photons, low loss microwave plasmonics analogous to optical plasmonics can be developed using superconducting thin films.

The fabrication of LHMs with magnetic response at $\mathrm{THz}$ and optical frequencies can be used for such application as compact cavities, tunable mirrors, security imaging, bio-molecular finger printing and remote sensing. Further unlike electrical networks and filters which primarily control voltage and current quantities in the frequency domain, transmission line metamaterials should also control the electromagnetic field distribution in the spatial domain (e.g. for deranging lenses, cloaks etc.).

Although the progress of cloaking device design is in the initial step, the underlying technology offers a unique approach to control electromagnetic wave propagation by complex media, controlling of electrons in inhomogeneous crystal systems, cold atoms in an optical lattices, radiation shielding, particle beam steering will have great potential in various radio frequency and optical application in the future.

\section{CONCLUSION:}

The past few years have illustrated the power of the metamaterial approach, because new material responses, some with no analog in usual materials, are now available for exploration. Pioneering experiments have been carried out in the radio frequency and microwave domains with millimeter or micrometer scale structures, but there is now a strong motivation for extending them to the optical domains using nanometer scale structures available with nanotechnology.

The present study provides a technical overview of the rapidly emerging metamaterial technology offering a wealth of new phenomena in optics and electromagnetism including negative refraction near field focusing, artificial magnetism, optical invisibility and the amplification of the evanescent waves. The article discusses the recent advances in the field and practical applications of possible devices. These artificial structures with electromagnetic response tailored to a particular objective such as the magnetic mirror [71] or an electromagnetic cloaking device [40] along with other several future devices which will pave the way to replace several conventional devices. The technology has potential applications, especially in optics, photonics, the science and technology utilizing lights, medicine and telecommunication. This article briefly presents all experimental and theoretical aspects of rapidly developing field ranging from basic science to applications and products.

\section{ACKNOWLEDGEMENTS:}

The present work has been carried out within the framework of the Associateship Scheme of the Abdus Salam International Centre for Theoretical Physics (ICTP), Trieste, Italy. The authors are grateful to ICTP for providing the necessary facilities and literature for the present work. MJ thanks late Prof. GK Johri for inspiration.

\section{REFERENCES:}

[1] Barber, DJ and Freestone, JC, An introduction of the origin of the colour of the Lycurgus cup by artificial transmission electron microscopy, 
Archaeometry, 1990, 32, 33-45.

[2] Wagner, FE, Haslbeck, S, Stievano, L, Calogero, S, Pankhurst, QA and Martinek, $\mathrm{P}$, Before striking gold in gold -ruby glass, Nature, 2000, 407, 691-692.

[3] Leonhardt, U, Optical Metamaterials invisibility cup, Nature photonics, 2007, 1, 207-208.

[4] Cui, TJ, Liu, R, and Smith, DR, Introduction to Metamaterials, In Metamaterials; Theory, Design and Application (eds. Cui, T. J., Smith, D.R., and Liu, R.), 2010, Springer, p. 1-19.

[5] Shamonina, E, and Solymar, L, Metamaterials: How the subject started, Metamaterials, 2008, 1, 12-18.

[6] Pendry, JB, Holden, AJ, Stewart, WJ, and Youngs, I, Extremely low frequency plasmons in metallic mesostructures. Phys. Rev. Lett., 1996, 76, pp. 4773-4776.

[7] Pendry, JB, Holden, AJ, Robbins, DJ and Stewart, WJ, Magnetism from Conductors and Enhanced Non-Linear Phenomena. IEEE Transactions on Microwave Theory and Techniques, 1999, 47 (11), 2075-2084.

[8] Cai, Wenshan and Shalaev, Vladimer, Optical Metamaterials: Fundamentals and Applications, 2010, ISBN 978-1-4419-11506, Springer, pp. 200.

[9] Johri, Manoj and Paudyal, Harihar, Left Handed Materials: A new paradigm in structured electromagnetic, 2010, IC/2010/015 available at http://publications.ictp.it.

[10] Freestone, Ian, Meeks, Nigel, Sax, Margaret, and Higgitt, Catherine, The Lycurgus Cup- A Roman Nanotechnology, Gold Bulletin, 2007, 40 (4), 270-277.

[11] Pekka, Alitalo and Tretyakov, Sergei, Electromagnetic Cloaking with Metamaterial. Materials Today, 2009, 12 (3), 22-29.

[12] Solymar, L and Shamonina, E, Waves in Metamaterials, Oxford University Press, New York (2009).

[13] Tretyakov, S, Analytical Modeling in Applied Electromagnetics, Artech House, Boston (2003).

[14] Marqués, R, Martín, F and Sorolla, M, Metamaterials with Negative Parameters: Theory, Design, and Microwave Applications, John Wiley and Sons, New York (2008).
[15] Bose, JC, On the Rotation of Plane of Polarization of Electric Waves by a Twisted Structure. Proc. Royal Soc. London, 1898, 63, 146.

[16] Lamb, H, On group velocity. Proc. London Math Soc., 1904, 1, 473-479.

[17] Pocklington, HC, Growth of a wave- group when the group velocity is negative. Nature, 1905, 71, 607-608.

[18] Lindell, IV, Sihvola, AH, and Kurkijarvi, J, Karl F Lindmann- the last Hertizian and a harbinger of electromagnetic chirality, IEEE Antenna Propag Mag, 1992, 34 (3), 24-30.

[19] Schuster, A, An Introduction to the theory of Optics (Edward Arnold, London), 1904.

[20] Mandelstam, LJ, Lectures given on Feb. 26, 1940 and May 5, 1944// Complete issue of works, vol. 5, Moscow, Izdatelstvo trudov AN SSSR, 1950.

[21] Tretyakov, SA, Research on negative refraction and backward wave media: A historical perspective, Negative refraction: revisiting electromagnetic from microwaves to optics, EPFL Latsis Symposium, Lausanne, 2005.

[22] Mandlshtam, L, Group velocity in a crystal lattice, ZET Fiziki, 1945, 15, 476-478. (in Russian).

[23] Malyuzhinets, GD, A note on the radiation principle, Zhurnal Tech. Fiziki, 1951, 21, 940-942 (in Russian).

[24] Sivukhin, DV, The energy of electromagnetic waves in dispersive media, Optica J Spectroskopiya, 1957, 3, 308-312.

[25] Kock, W, Metallic Delay Lenses. Bell System Technical J., 1948, 27, 58.

[26] Pafomov, VE, Transition radiation and Cherenkov radiation. Soviet Physics JEPT, 1959, 9, 1321.

Hutter, RGE, Beam and wave electronics in microwave tubes, Von Nostrand Reinhold Princeton, NJ, 1960, 220-230.

Altman, JL, Microwave Circuits, Von Nostrand Reinhold Princeton, NJ, 1964, 304. Silin, RA and Suzonov,VP, Slow wave 
structures, Moscow, 1966 (in Russian).

[30] Silin, RA, Waveguiding properties of two dimensional periodical slow wave system, Voprosy Radioelektronoki, Electronika, 1959, 4, 11-33.

[31] Dollin, LS, Equation for the correlation functions of a wave beam in a randomly inhomogeneous medium, IZV- VUZOR Radiofizika, 1961, 486-491 (in Russian).

[32] Veselago, VG, The Electrodynamics of Substances with Simultaneously Negative Values of $\varepsilon$ and $\mu$. Sov. Phys. Usp., 1968, 10, 509-514.

[33] Kerker, MJ, Invisible bodies, J. Opt. Soc. Am., 1975, 65, 376-379.

[34] Walser, RM, 1999, http://en.wikipedia.org/ wiki/Metamaterial.

[35] Smith, DR, Padilla, WJ, Nemat-Nasser, SC and Schultz, S, Composite Medium with Simultaneously Negative Permeability and Permittivity. Phys. Rev. Lett., 2000, 84, 4184-4187.

[36] Shelby RA, Smith, DR, and Schultz, S, Experimental Verification of a Negative Index of Refraction. Science, 2001, 292, 7779 .

[37] Tassin, P, Van der Sande G and Veretennicoff, I, Left Handed materials: the key to subwavelength resolution?. Proc. IEEE/ LEOS , 2004, p. 41-44.

[38] Smith, DR, Pendry, JB and Wiltshire, MCK, Metamaterials and Negative refractive Index. Science, 2004, 305, 788-790.

[39] Pendry, JB, Negative Refraction Makes a Perfect Lens. Phys. Rev. Lett., 2000, 85, 3966-3969.

[40] Pendry JB, Schurig, D and Smith, DR, Controlling electromagnetic fields. Science, 2006, 312, 1780-1782.

[41] Schurig, D, Mock, JJ, Justice BJ, Metamaterial electromagnetic cloak at microwave frequencies, Science, 2006, 314, 977-980.

[42] Liu, R, Cui, TJ, Huang, D, Zhao, B and Smith DR, Broadband ground - plane cloak,
Science, 2009, 323, 366.

[43] Valentine, J, Li, J, Zentrgraf, T, Bartal, G and Zhang X, An optical cloak made of dielectric, Nature Materials 2009, 8, 568-571.

[44] Gabrielli, L H, Cardenar, J, Poitras, C B and Lipson, M, Nat. Photonics, 2009, 3, 461.

[45] Lee, JH, Blair, J, Tamma, VA, Wu, Q, Rhee, SJ, Summers, CJ and Park, W, Direct visualization of optical frequency invisibility cloak based on silicon nanorod array, Optical Express, 2009, 17 (15), 12922-12928.

[46]

Smolyaninov, L I, Smolyaninova, V M, Kildishev, A V and Shalaev, V M, Phys. Rev. Lett, 2009, 102, 213901.

[47] Ergin, Tolga, Stenger, N, Brenner, P, Pendry, $\mathrm{J}$ B, and Wegener, M, Three dimensional invisibility cloak at optical wavelengths, Science, 2010, 328, 337-339.

[48] Ramakrishna, SA, Physics of negative index materials, Rep. Prog. Phys., 2005, 68, 449521.

[49] Pendry, J. B., Focus issue: negative reflection and metamaterials - introduction. doi:10.1364/OE.11.000639, Opt. Express, 2003, 11, 639.

[50] Shuang, Zhang, Dentcho, A. Genov, Cheng Sun and Xiang Zhang, Cloaking of matter waves, Physical Review Letters, 2008, 100, 123002.

[51] Alu, A. and Engheta, N., Plasmonic and metamaterial cloaking: physical mechanisms and potentials, J. Opt A, 2008, 10, 093002.

[52] Di Gennaro, E., Parimi, P.V., Lu, W.T., Sridhar, S., Deror, J.S., and Turchinetz, B., 2005, Phys. Rev. B, 72, 033110.

[53] Yen, T.J., Padilla, W.J., Fang, N., Vier, D.C., Smith, D.R., Pendry, J.B., Basov, D.N. and Zhang, X., Terahertz magnetic response from artificial materials. 2004, Science, 303, 1494.

[54] Kante, B., Ourir, A., Burokur, S.N., Gadot, F. and Lustrac, A., Matematerial for optical and radio communication. 2008, Comptes Rendus Physique, 9, 31-40.

[55] Silveirinha, M., and Engheta, N., Tunneling of electromagnetic energy through subwavelength channels and bends using 
epsilon near zero materials. 2006, Phys. Rev. Lett., 97, 157403.

[56] Edwards, Brian, Alu, A., Young, M.E., Silveirinha, M. and Engheta, N., Experimental verification of epsilon-nearzero metamaterial coupling and energy squeezing using a microwave waveguide. 2008, Phys. Rev. Lett., 100, 033904.

[57] Enoch, S., Taveb, G., Sabouroux, P., Guerin, N. and Vincent, P., A metamaterial for directive emission. 2002, Phys. Rev. Lett., 89, 213902.

[58] Eleftheriades, George V., EM transmissionline metamaterials. 2009, Materials Today, 12 (3), 30-41.

[59] Tiwari, Akhilesh, Fontaine, Jean-Pierre and Johri, Manoj, The study of Negative refractive Index metamaterials in the presence of nanophotonics. Proc. $2^{\text {nd }}$ National Conf. on nanomaterials, 2009, ISBN 978-93-8004361-6, p. 291.

[60] John, S., Strong localization of photons in certain disordered dielectric superlattices. Phys. Rev. Lett., 1987, 58, 2486-2489.

[61] Yablonovitch, E., Inhibited spontaneous emission in Solid State Physics and Electronics, Phys. Rev. Lett., 1987, 58, 2327-2335.

[62] Chan, C. T., Ho, K. M. and Soukolis, C. M., Existence of a photonic gap in periodic dielectric structures. Phys. Rev. Lett., 1990, 65, 3152-3155.

[63] Pendry, JB and Smith, DR, Reversing light with negative refraction. Physics Today, 2004, 57 (6), 37-43.

[64] Yablonovitch, E., Gmitter, T. and Leung, K.,
Photonic band structure: The face-centeredcubic case employing nonspherical atoms. Phys. Rev. Lett., 1991, 67, 2295-2298.

[65] Johri, G. K., Tiwari, A., Johri, M. and Yoshino, $\mathrm{K}$., Theoretical study of temperature tuning and anisotropy of liquid crystal infiltrated synthetic opal as photonic crystal. Jpn. J. Appl. Phys., 2001, 40, 4565-4569.

[66] Johri, G. K., Tiwari, A., Saxena, S. and Johri, M., Existence of photonic band gap and underlying physical process. Mod. Phys. Lett. B, 2001, 15, 529-534.

[67] Johri, G. K., Tiwari, A., Saxena, S., Sharma, R., Srivastava, K., and Johri, M., Theoretical study of relative width of photonic band gap for the 3-D dielectric structure. Pramana, 2002, 58, 563.

[68] Johri, G.K., Johri, M., Tiwari, A. and Yoshino, $\mathrm{K}$., Dielectric material as photonic crystal and formulation of Lambshift using anisotropic model. IEEE TDEI, 2004, 11, 184-189.

[69] Johri, M., Ahmed, Y.A. and Bezboruah, T., Photonic band gap materials; technology, application and challenges. Current Science, 2007, 92 (10) 1361-1365.

[70] Anlage, Steven M., The physics and applications of superconducting metamaterials, Journal of Optics, 2010, 1-17 (http://arxiv.org/ftp/arxiv/ papers/1004/1004.3226.pdf).

[71] Fedotov, V.A., Rogacheva, A.V., Zheludev, N.I., Miadyonov, P.L. and Prosvirnin, S.L., Morror that does not change the phase of reflected waves, 2006, Appl. Phys. Lett., 88, 091119. 